\title{
EDITORIAL
}

\section{DIVERSIDAD EN LA MULTICULTURALIDAD DEL CONOCIMIENTO CIENTÍFICO. LAS CIENCIAS BÁSICAS Y LA INGENIERÍA PARA LA CONSTRUCCIÓN DE SABERES EN DIFERENTES CONTEXTOS}

El número 25 de nuestra publicación refleja diferentes aportes enfocados en la construcción del conocimiento para el campo de las ciencias básicas e ingeniería. Dentro de los aportes brindados por los autores se encuentran aspectos de interés tales como el juego como estrategia para el fortalecimiento de habilidades cognitivas en estudiantes que se encuentran con pérdida académica en espacios académicos de la ingeniería. Este manuscrito refleja la mirada desde la parte psicológica de los estudiantes en este objeto de estudio para la formación en ingeniería. En la misma medida, se resalta el aporte de dos manuscritos referidos a la energía en cuanto a su caracterización e implicaciones; cada uno de ellos refleja los aportes significativos dados por los autores frente a esta temática vigente y relevante para las problemáticas actuales de nuestra sociedad colombiana.

Desde del campo ambiental se presenta un manuscrito enfocado en la evaluación de la calidad del agua niebla del municipio de Choachí, Cundinamarca, que aporta en la transformación de las comunidades como campo fundamental de la investigación de la Corporación Universitaria Minuto de Dios - UNIMINUTO, lo cual coadyuva a impactar positivamente en la calidad de vida de los residentes aledaños, la puesta en marcha en el cambio de paradigmas, la disminución de los minerales y el aumento de materiales derivados de la porcicultura en los cuerpos de agua limpias (derivadas del agua niebla) como fuente de consumo potable para los habitantes de la región.

En el marco de la Ingeniería Civil se presenta el aporte de un manuscrito que establece las diferencias cuantitativas entre sistemas constructivos in situ y prefabricados como soporte para la toma de decisiones para el sector productivo, donde se examinan los parámetros de diseño y construcción de entre piso de los sistemas tradicionales prefabricados. Se considera un aporte significativo para la comunidad científica ya que desde la Ingeniería Civil los trabajos en su mayoría han estado centrados en los aspectos de tipo cualitativo respecto de caracterización y evaluación de materiales. De igual forma, se vislumbra este aporte como una herramienta útil para el sector productivo en la toma de decisiones.

Desde la perspectiva agroecológica, aparece el aporte a las comunidades campesinas del municipio de Guayabal de Síquima, Cundinamarca, el cual permite las mejoras en la calidad de vida, a través de la aplicación de técnicas de agriculturas alternativas y el empoderamiento del campesino para que sea este el gestor y protagonista de los cambios generados en su sistema productivo y su calidad de vida, utilizando metodologías de investigación social de tipo participativo, en pro del desarrollo endógeno de dicha comunidad. Este proceso presentó diversos niveles de aceptación y diversos porcentajes de adopción, adaptación y utilización de las técnicas transferidas.

Desde el campo de la tecnología, uno de los aportes se centra en la implementación de una plataforma de telefonía IP a través de red wifi utilizando software libre, lo cual permitió realizar llamadas entre extensiones móviles aprovechando el uso de la red en una compañía determinada en pequeñas y medianas empresas.

Finalmente, encontramos el aporte centrado en el sector aeronáutico, que aborda la familiarización y calificación de aeropuertos en la industria aeronáutica, en el ámbito nacional e internacional, para ser aplicados en SATENA. En el mismo sentido, explora las herramientas utilizadas y los elementos seleccionados para el diseño del programa, con el fin de prevenir accidentes. Este escrito se llevó a cabo por medio de una revisión documental para observar los análisis de los factores contribuyentes en el tema y se refleja como una herramienta útil para el campo de la Ingeniería Industrial y procesos logísticos.

Julio César Rivera Rodríguez 